\title{
A Lower Bound for the Optimal Crossing-Free Hamiltonian Cycle Problem*
}

\author{
Ryan B. Hayward \\ School of Computer Science, McGill University, 805 Sherbrooke St. West, Montreal, \\ Quebec, Canada H3A 2K6
}

\begin{abstract}
Consider a drawing in the plane of $K_{n}$, the complete graph on $n$ vertices. If all edges are restricted to be straight line segments, the drawing is called rectilinear. Consider a Hamiltonian cycle in a drawing of $K_{n}$. If no pair of the edges of the cycle cross, it is called a crossing-free Hamiltonian cycle (cfhc). Let $\Phi(n)$ represent the maximum number of cfhc's of any drawing of $K_{n}$, and $\Phi(n)$ the maximum number of cfhc's of any rectilinear drawing of $K_{n}$. The problem of determining $\Phi(n)$ and $\bar{\Phi}(n)$, and determining which drawings have this many cfhc's, is known as the optimal cfhc problem. We present a brief survey of recent work on this problem, and then, employing a recursive counting argument based on computer enumeration, we establish a substantially improved lower bound for $\Phi(n)$ and $\Phi(n)$. In particular, it is shown that $\bar{\Phi}(n)$ is at least $k \times 3.2684^{n}$. We conjecture that both $\Phi(n)$ and $\bar{\Phi}(n)$ are at most $c \times 4.5^{n}$.
\end{abstract}

\section{A Survey of the Optimal Crossing-Free Hamilton Cycle Problem}

Let $K_{n}$ be the complete graph on $n$ vertices. All drawings in this paper are assumed to be drawn in the plane. If all the edges of a drawing of a graph are restricted to be straight line segments, the drawing is said to be rectilinear. By a crossing of a drawing we mean a pair of edges which intersect in the drawing. A Hamiltonian cycle of a graph is a cycle that visits each vertex of the graph exactly once. Consider a particular Hamiltonian cycle in a drawing of $\boldsymbol{K}_{n}$. If the cycle includes no crossings, it is called a crossing-free Hamiltonian cycle, or a cfhc for short. Let $\Phi(n)$ (and respectively $\bar{\Phi}(n)$ ) represent the maximum number of cfhc's of any drawing (respectively rectilinear drawing) of $K_{n}$. The optimal $c f h c$ problem is to determine $\Phi(n)$ and $\bar{\Phi}(n)$, and to determine which drawings

* This research, part of which was conducted at Queen's University, was supported by an N.S.E.R.C. postgraduate scholarship. 

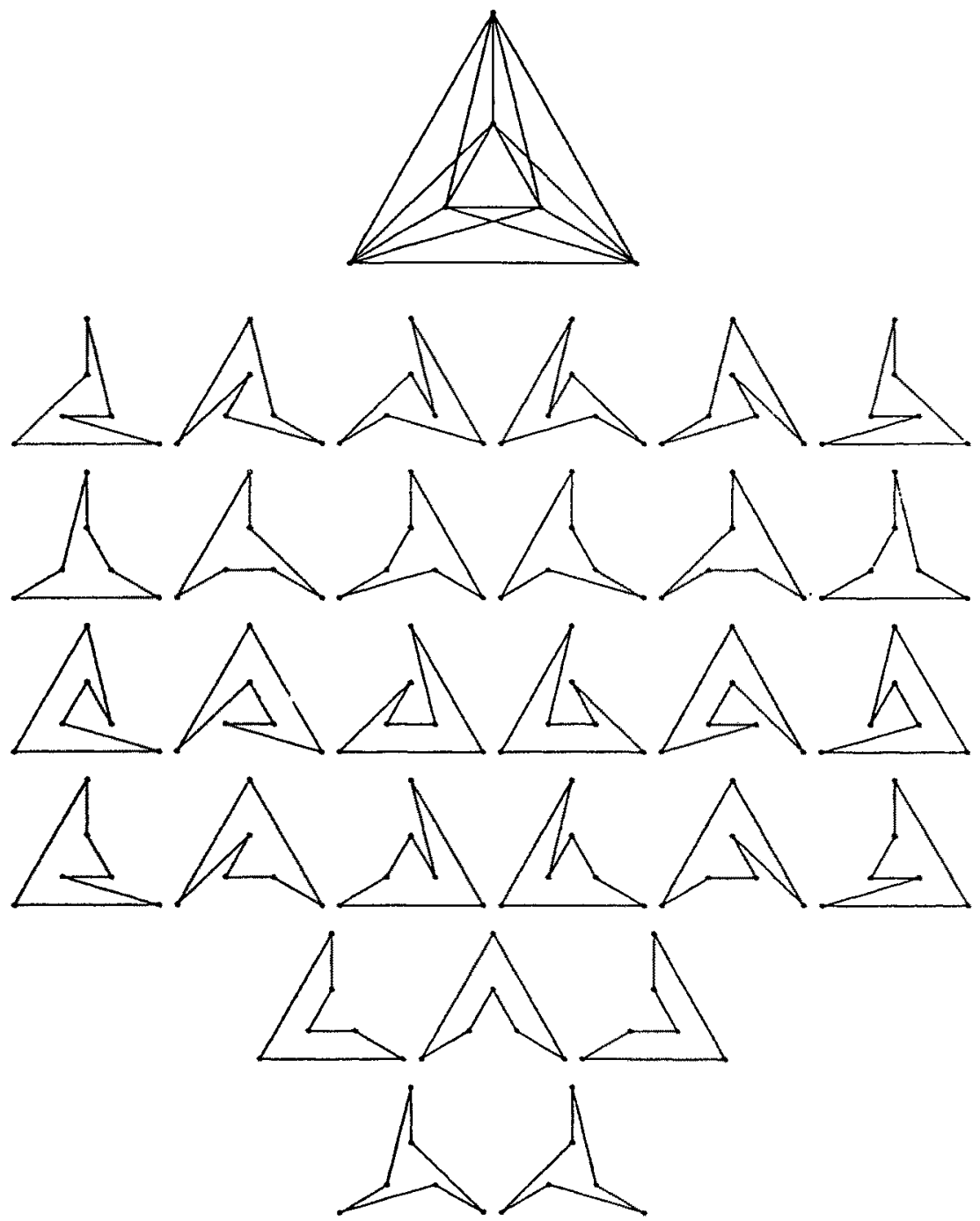

Fig. 1. A drawing of $K_{6}$ with $29 \mathrm{chc}$ 's.

of $K_{n}$ have $\Phi(n)$ (respectively $\bar{\Phi}(n)$ ) cfhc's. Such drawings will be referred to as cfhc-optimal (respectively rectilinear cfhc-optimal) drawings. Figure 1 shows a drawing of $K_{6}$ which is both cfhc-optimal and rectilinear cfhc-optimal. The drawing has 29 cfhc's; the other 91 Hamiltonian cycles all have at least one crossing.

Let $\nu(n)$ (respectively $\bar{\nu}(n)$ ) refer to the minimum number of crossings of any drawing (respectively rectilinear drawing) of $K_{n}$. The optimal crossing problem, also known as the crossing number problem, is to determine the values of $\nu(n)$ and $\bar{\nu}(n)$, and to find which drawings attain this number of crossings. The optimal 
crossing problem is related to the optimal cfhc problem in that, in general, drawings with fewer crossings have more cfhc's. However, this is not always the case (e.g., see [H]). Although the optimal crossing problem has been extensively studied (see [EG] or [G]), exact values for $\nu(n)$ and $\bar{\nu}(n)$ are not known for $n>10$.

The optimal cfhc problem was first explored by Newborn and Moser [NM]. They were able to determine $\Phi(n)$ and $\bar{\Phi}(n)$ exactly for $n$ from 3 to 6 , and established lower bounds for other small values of $n$. Later we extended this list of lower bounds [H]. The following is a list of the current best lower bounds for $\Phi(n)$ and $\bar{\Phi}(n)$, for $n$ up to 13 (values for $n$ up to 8 were established in [NM], all others are taken from $[\mathrm{H}])$ :

Best known lower bounds

\begin{tabular}{cccccccccccc}
\hline $\mathrm{n}$ & 3 & 4 & 5 & 6 & 7 & 8 & 9 & 10 & 11 & 12 & 13 \\
\hline$\Phi(n)$ & 1 & 3 & 8 & 29 & 92 & 339 & 1252 & 4956 & 18383 & 75231 & 306446 \\
$\bar{\Phi}(n)$ & 1 & 3 & 8 & 29 & 96 & 399 & 1461 & 6354 & 24687 & 110162 & 446798 \\
\hline
\end{tabular}

The first bounds for $\Phi(n)$ or $\bar{\Phi}(n)$ for arbitrary $n$ were established by Newborn and Moser, who showed that

$$
\frac{3}{20} \times 10^{n / 3} \leq \bar{\Phi}(n) \leq 2 \times 6^{n-2} \times\left[\frac{n}{2}\right], \quad \text { where } \quad 10^{1 / 3} \doteq 2.1544 .
$$

The upper bound was substantially improved by Ajtai et al. [ACNS], who showed that every planar drawing of any graph with $n$ vertices contains at most $10000000000000^{n}$ crossing-free subgraphs. Thus both $\Phi(n)$ and $\bar{\Phi}(n)$ are exponential in $n$.

The lower bound was first improved by Akl [A], who showed that $d_{n}<\bar{\Phi}(n)$, where $d_{n}$ is asymptotically $k \times(5+3 \times \sqrt{5})^{n / 3}$, with $k$ a constant and $(5+3 \times$ $\sqrt{5})^{1 / 3} \doteq 2.2707$.

In this paper, generalizing Akl's approach, we show how the lower bound can be substantially improved by counting a subset of the cfhc's of a certain drawing $\mathrm{TS}_{n}$ of $K_{n}$. We prove that $f_{n}<\bar{\Phi}(n)$, where $f_{n}$ is asymptotically $k \times 3.2684^{n}$.

\section{An Improved Lower Bound for $\overline{\boldsymbol{\Phi}}(\boldsymbol{n})$}

In this section we describe a certain rectilinear drawing $\operatorname{TS}_{n}$ of $K_{n}$, and then count a subset of its cfhc's. This gives a new lower bound for $\bar{\Phi}(n)$.

\subsection{A Description of the Drawing $\mathrm{TS}_{n}$}

The "TS" in TS $_{n}$ is mnemonic for "trilateral spiral". Roughly speaking, the vertices of $\mathrm{TS}_{n}$ can be thought of as resting on three gently spiralled arcs emanating from the origin. 
More precisely, let $\operatorname{arc} A$ be the arc of the circle centered at the point in the plane with Cartesian coordinates $(x, 2)$ and joining (in clockwise order) the points $(0,1)$ and $(0,3)$, where $x \geq 7 / \sqrt{3}$. Arcs $B$ and $C$ are formed by rotating $\operatorname{arc} A$ respectively 120 and $240^{\circ}$ clockwise about the origin, namely the point $(0,0)$. Place vertices $1,4,7, \ldots$ on arc $A$, vertices $2,5,8, \ldots$ on arc $B$, and vertices $3,6,9, \ldots$ on arc $C$, so that if $v$ and $w$ are on the same arc, and $v<w$, then $v$ is closer to the origin than $w$ (see Fig. 2). Figures 1,3, and 4 show drawings of $\mathrm{TS}_{6}, \mathrm{TS}_{9}$, and $\mathrm{TS}_{12}$, respectively.

The reason for choosing $x$ as described above is to ensure that the line segment joining the far end of arc $A$ to the near end of arc $C$ does not intersect arc $A$ in any other point. In fact, the arcs are constructed so that any line segment joining points on two different arcs intersects each of the two arcs in exactly one point, and does not intersect the third arc.

Let

$$
a=\left[\frac{n+2}{3}\right], \quad b=\left[\frac{n+1}{3}\right], \quad c=\left[\frac{n}{3}\right]
$$

and relabel vertices $1,4, \ldots, 3 a-2$ as $A_{1}, A_{2}, \ldots, A_{a}$, vertices $2,5, \ldots, 3 b-1$ as $B_{1}, B_{2}, \ldots, B_{b}$, and vertices $3,6, \ldots, 3 c$ as $C_{1}, C_{2}, \ldots, C_{c}$. Then the following
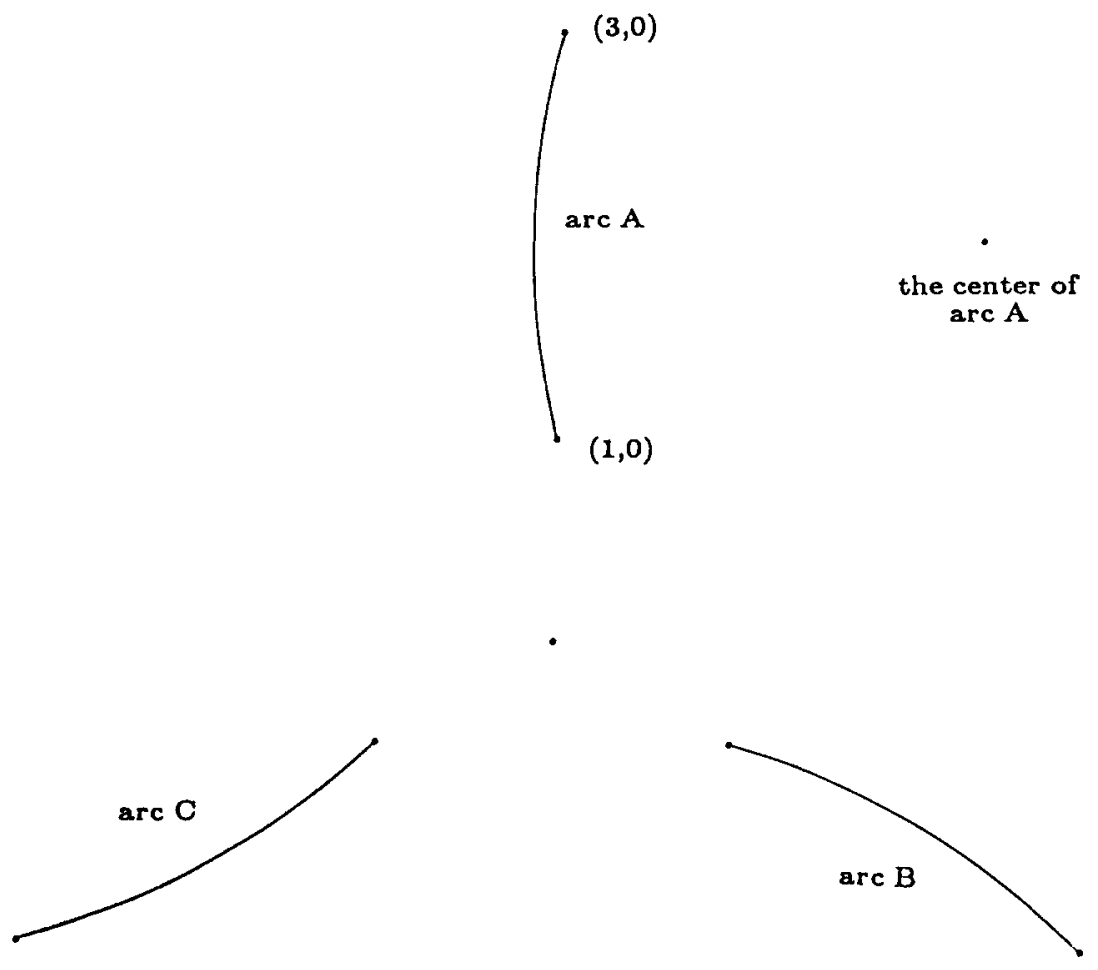

Fig. 2. Template arcs for $\mathrm{TS}_{n}$. 


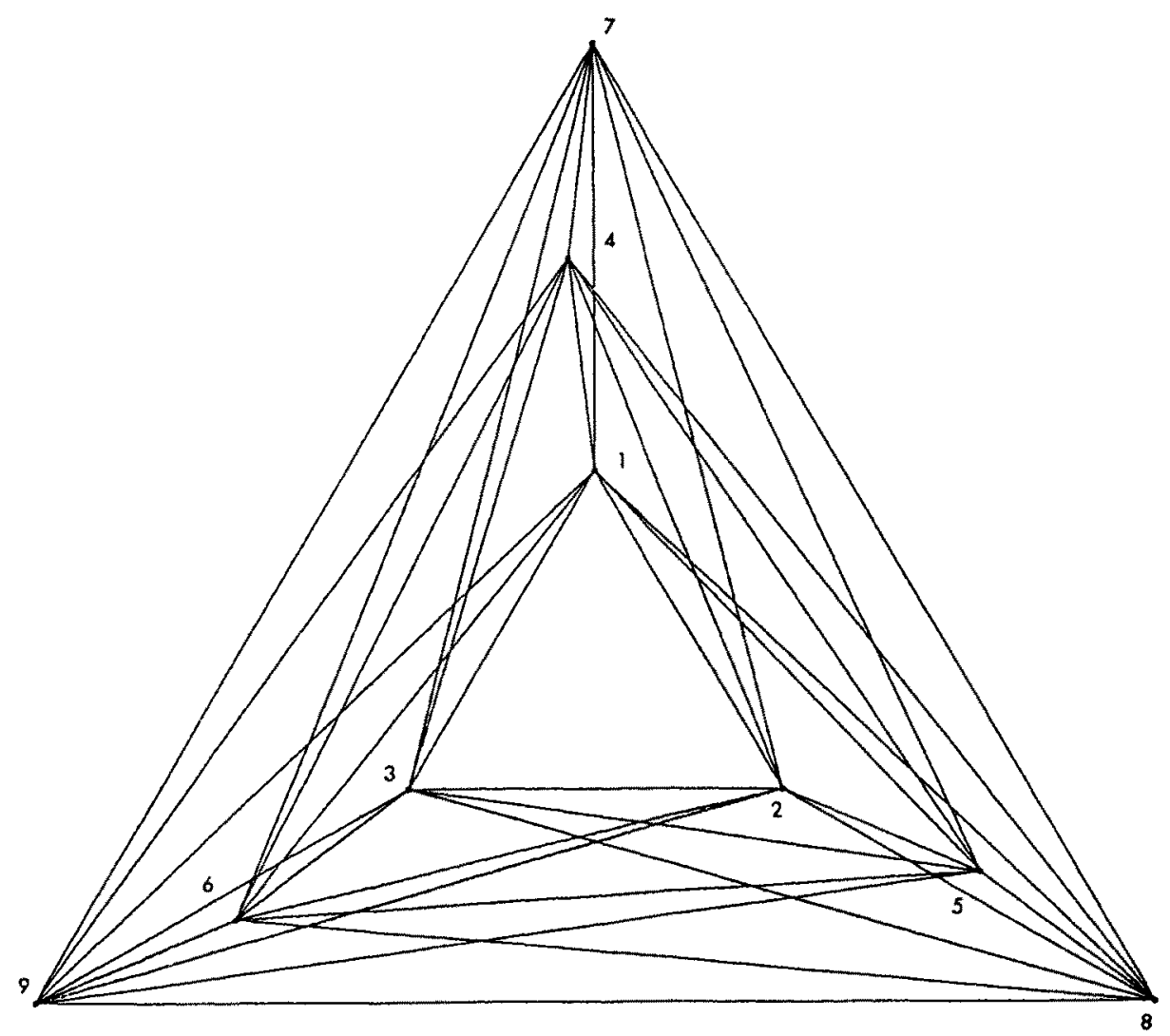

Fig. 3. The drawing $\mathrm{TS}_{9}$.

is a description of all crossings of $\mathrm{TS}_{n}$ :

(1) $\left(A_{i}, A_{k}\right)\left(A_{j}, A_{m}\right)$ for $1 \leq i<j<k<m \leq a$,

(2) $\left(B_{i}, B_{k}\right)\left(B_{j}, B_{m}\right)$ for $1 \leq i<j<k<m \leq b$,

(3) $\left(C_{i}, C_{k}\right)\left(C_{j}, C_{m}\right)$ for $1 \leq i<j<k<m \leq c$,

(4) $\left(A_{i}, B_{k}\right)\left(A_{j}, B_{m}\right)$ for $1 \leq i<j \leq a, 1 \leq k<m \leq b$,

(5) $\left(B_{i}, C_{k}\right)\left(B_{j}, C_{m}\right)$ for $1 \leq i<j \leq b, 1 \leq k<m \leq c$,

(6) $\left(C_{i}, A_{k}\right)\left(C_{j}, A_{m}\right)$ for $1 \leq i<j \leq c, 1 \leq k<m \leq a$,

(7) $\left(A_{i}, A_{k}\right)\left(A_{j}, B_{m}\right)$ for $1 \leq i<j<k \leq a, \quad 1 \leq m \leq b$,

(8) $\left(B_{i}, B_{k}\right)\left(B_{j}, C_{m}\right)$ for $1 \leq i<j<k \leq b, \quad 1 \leq m \leq c$,

(9) $\left(C_{i}, C_{k}\right)\left(C_{j}, A_{m}\right)$ for $1 \leq i<j<k \leq c, 1 \leq m \leq a$. 


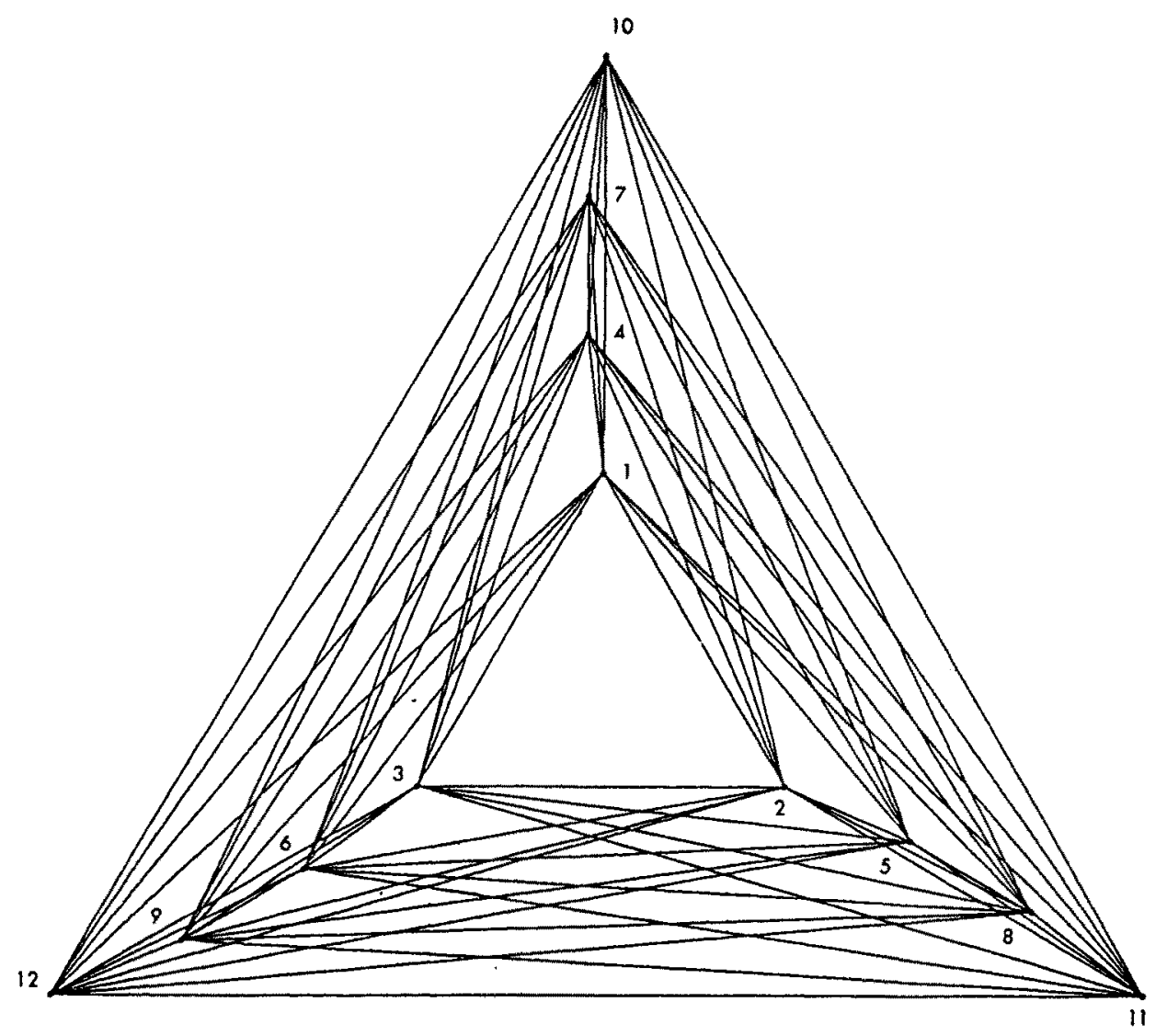

Fig. 4. The drawing $\operatorname{TS}_{12}$.

The number of crossings is

$$
\left(\begin{array}{l}
a \\
4
\end{array}\right)+\left(\begin{array}{l}
b \\
4
\end{array}\right)+\left(\begin{array}{l}
c \\
4
\end{array}\right)+\left(\begin{array}{l}
a \\
2
\end{array}\right)\left(\begin{array}{l}
b \\
2
\end{array}\right)+\left(\begin{array}{l}
b \\
2
\end{array}\right)\left(\begin{array}{l}
c \\
2
\end{array}\right)+\left(\begin{array}{l}
c \\
2
\end{array}\right)\left(\begin{array}{l}
a \\
2
\end{array}\right)+\left(\begin{array}{l}
a \\
3
\end{array}\right) b+\left(\begin{array}{l}
b \\
3
\end{array}\right) c+\left(\begin{array}{l}
c \\
3
\end{array}\right) a
$$

where $\left(\begin{array}{l}x \\ y\end{array}\right)$ is defined as 0 if $x<y$. Thus, the total number of crossings of $\operatorname{TS}_{n}$ is

$$
\begin{gathered}
\frac{11 n^{4}-90 n^{3}+225 n^{2}-162 n}{648} \\
\frac{11 n^{4}-90 n^{3}+249 n^{2}-290 n+120}{648} \\
\frac{11 n^{4}-90 n^{3}+249 n^{2}-250 n+48}{648}
\end{gathered}
$$

for $n$ congruent to $0,1,2(\bmod 3)$, respectively. 


\subsection{Counting cfhc's of $\mathrm{TS}_{n}$}

Let $\mathrm{cfhc}\left(\mathrm{TS}_{n}\right)$ represent the number of cfhc's of $\mathrm{TS}_{n}$. We are unable to determine cfhc $\left(\operatorname{TS}_{n}\right.$ ) explicitly for arbitrary $n$. However, by counting a proper subset of the cfhc's of TS $_{n}$, we have established a lower bound for cfhc $\left(\mathrm{TS}_{n}\right)$, which gives an improved lower bound for $\bar{\Phi}(n)$.

Our counting argument is inductive, and relies on the fact that in any drawing of $\mathrm{TS}_{n}$, any consecutive set of $r$ vertices induces a drawing isomorphic to $\mathrm{TS}_{r}$ (two drawings of $K_{n}$ are isomorphic if the vertices of one can be relabeled so that both drawings have the same set of crossings). Thus it follows that in a drawing of $\mathrm{TS}_{n+k}$, the drawing induced by vertices 1 to $n$ is isomorphic to $\mathrm{TS}_{n}$. We will count cfhc's of TS $_{n+k}$ by counting cfhc's of TS ${ }_{n}$, and then enumerating various ways in which cfhc's of $\mathrm{TS}_{n}$ give rise to cfhc's of $\mathrm{TS}_{n+k}$.

We classify each cfhc of TS $_{n}$ according to which of the three outermost (convex hull) edges and which of the three innermost edges the cfhc contains. In $\operatorname{TS}_{n}, X, Y$, and $Z$ will represent, respectively, the edges $(n-2, n-1),(n-1, n)$, and $(n-2, n)$ and $x, y$, and $z$ will represent the edges $(1,2),(2,3)$, and $(1,3)$. We will use $\gamma$ to represent cfhc's. Thus a $\gamma(X, n)$ will represent a cfhc of $\operatorname{TS}_{n}$ that includes the edge $(n-2, n-1)$ but neither edge $(n-1, n)$ nor $(n-2, n)$. A $\gamma(y z, n)$ will represent a cfhc of $\operatorname{TS}_{n}$ that includes the edges $(2,3)$ and $(1,3)$ but not edge $(1,2)$. We will ignore cfhc's which contain all or none of either the outermost or innermost edges.

We create cfhc's of $\mathrm{TS}_{n+k}$ by starting with a cfhc of $\mathrm{TS}_{n}$ on vertices 1 to $n$, removing either one or two of its outermost edges, and then joining the resulting crossing-free path to vertices $n+1$ to $n+k$. For $k=1$ and 2 we enumerate by hand all the possible ways in which this can be done. For $k \geq 3$ we show how this can be done in a more systematic way (and in a way which allows for computer enumeration).

2.2.1. Case $k=1$ : creating cfhc's of $\mathrm{TS}_{n+1}$ from cfhc's of $\mathrm{TS}_{n}$. Figure 5 shows all nine ways in which cfhc's of TS $_{n}$ give rise to cfhc's of TS $_{n+1}$ upon removal of an outermost edge. (Only vertices $n-2$ to $n+1$ of TS $_{n+1}$ are shown in Fig. 5 . The dashed line in the figures represents that part of the $\mathrm{cfhc}$ which visits vertices 1 to $n-3$.) In particular,

each $\gamma(X, n)$ gives rise to a $\gamma(Z, n+1)$,

each $\gamma(Y, n)$ gives rise to a $\gamma(Y Z, n+1)$,

each $\gamma(Z, n)$ gives rise to a $\gamma(Y, n+1)$,

each $\gamma(Y Z, n)$ gives rise to a $\gamma(Y Z, n+1)$ and a $\gamma(X Y, n+1)$,

each $\gamma(Z X, n)$ gives rise to a $\gamma(Y, n+1)$ and a $\gamma(Z, n+1)$,

each $\gamma(X Y, n)$ gives rise to a $\gamma(Y Z, n+1)$ and a $\gamma(Z X, n+1)$.

For $\Omega=X, Y, Z, Y Z, Z X, X Y$, let cfhc $(\Omega, n)$ represent the number of $\gamma(\Omega, n)$, and let $t_{n}$ be the six element vector whose components are cfhc $(\Omega, n)$. Then we have shown that

$$
t_{n+1} \geq N_{1} \times t_{n}
$$



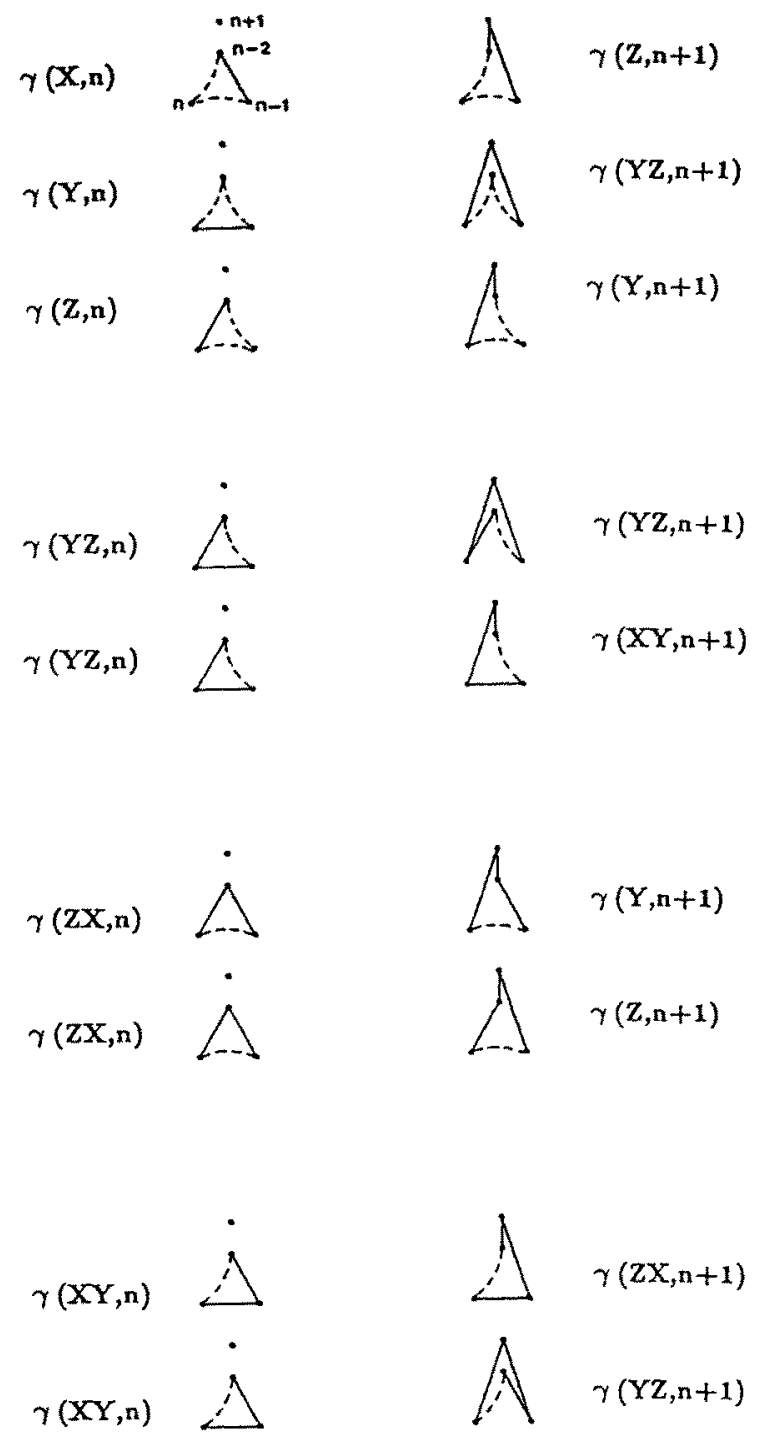

Fig. 5. Creating cfhc's of $\mathrm{TS}_{n+1}$ from chc's of $\mathrm{TS}_{n}$.

where

$$
N_{1}=\left[\begin{array}{llllll}
0 & 0 & 0 & 0 & 0 & 0 \\
0 & 0 & 1 & 0 & 1 & 0 \\
1 & 0 & 0 & 0 & 1 & 0 \\
0 & 1 & 0 & 1 & 0 & 1 \\
0 & 0 & 0 & 0 & 0 & 1 \\
0 & 0 & 0 & 1 & 0 & 0
\end{array}\right]
$$


From (1) it follows that cfhc $\left(\mathrm{TS}_{n}\right)$ is asymptotically at least $c \times r_{1}^{n}$, where $c$ is some constant and $r_{1}$ is the dominant eigenvalue of $N_{1}$, namely (to four decimal places) 1.8124 .

2.2.2. Case $k=2$ : creating cfhc's of $\mathrm{TS}_{n+2}$ from cfhc's of $\mathrm{TS}_{n}$. Figure 6 shows all ways in which cfhc's of $\mathrm{TS}_{n+2}$ are created from cfhc's of $\mathrm{TS}_{n}$. (Only vertices

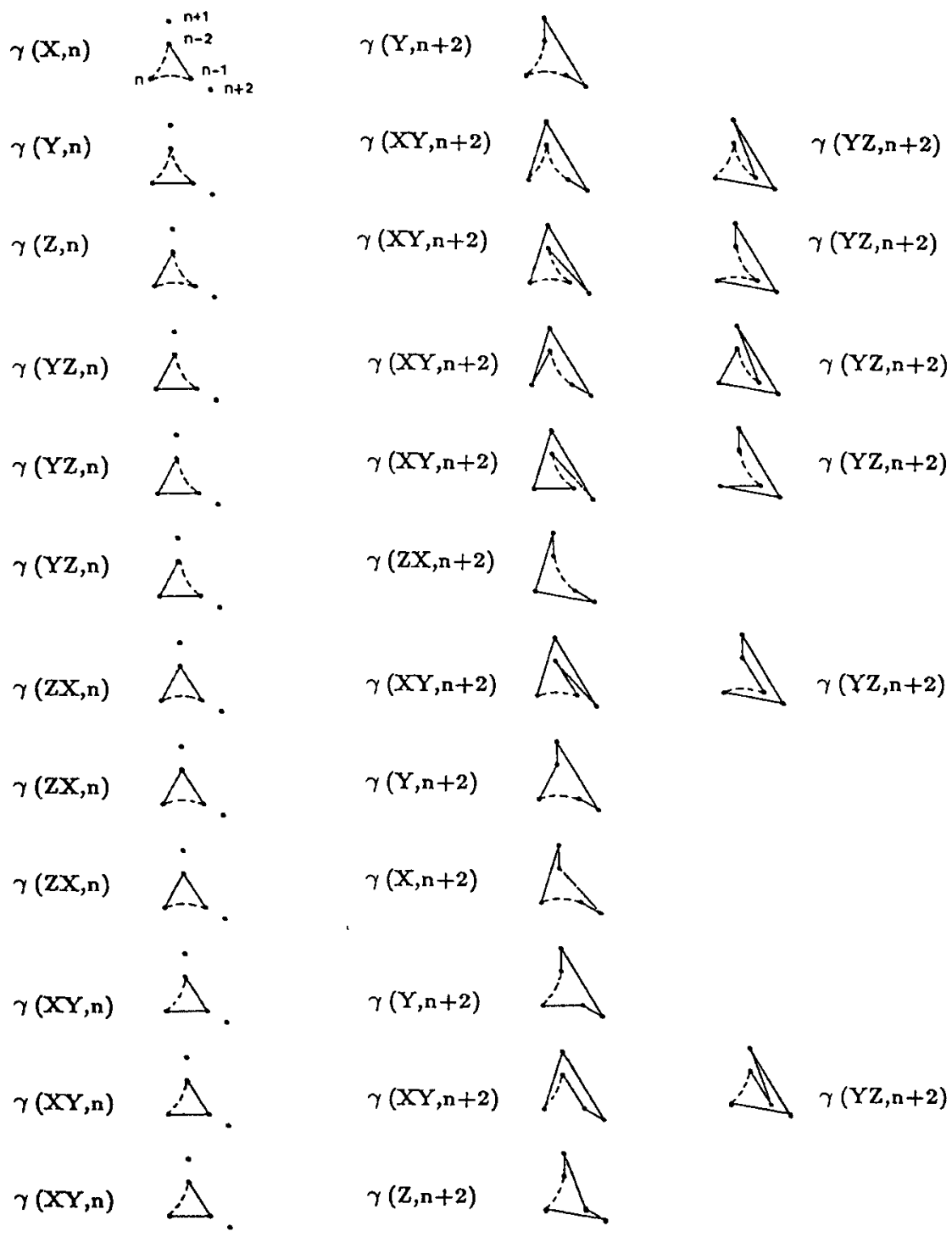

Fig. 6. Creating cfhc's of $\mathrm{TS}_{n+2}$ from cfhc's of $\mathrm{TS}_{n}$. 
$n-2$ to $n+2$ of $\mathrm{TS}_{n+2}$ are shown in Fig. 6.) In particular,

each $\gamma(X, n)$ yields a $\gamma(Y, n+2)$,

each $\gamma(Y, n)$ yields a $\gamma(Y Z, n+2)$ and a $\gamma(X Y, n+2)$,

each $\gamma(Z, n)$ yields a $\gamma(Y Z, n+2)$ and a $\gamma(X Y, n+2)$,

each $\gamma(Y Z, n)$ yields two $\gamma(Y Z, n+2)$, a $\gamma(Z X, n+2)$, and two $\gamma(X Y, n+2)$, each $\gamma(Z X, n)$ yields a $\gamma(X, n+2)$, a $\gamma(Y, n+2)$, a $\gamma(Y Z, n+2)$, and a $\gamma(X Y, n+2)$,

each $\gamma(X Y, n)$ yields a $\gamma(Y, n+2)$, a $\gamma(Z, n+2)$, a $\gamma(Y Z, n+2)$, and a $\gamma(X Y, n+2)$.

This information is summarized in matrix form as

$$
t_{n+2} \geq N_{2} \times t_{n},
$$

where

$$
N_{2}=\left[\begin{array}{llllll}
0 & 0 & 0 & 0 & 1 & 0 \\
1 & 0 & 0 & 0 & 1 & 1 \\
0 & 0 & 0 & 0 & 0 & 1 \\
0 & 1 & 1 & 2 & 1 & 1 \\
0 & 0 & 0 & 1 & 0 & 0 \\
0 & 1 & 1 & 2 & 1 & 1
\end{array}\right]
$$

Finally, note that if we count chh's of $\mathrm{TS}_{n+2}$ created by either adding two vertices to a cfhc of $\mathrm{TS}_{n}$ (as just described), or by twice adding a single vertex (as described in Case $k=1$ above), then we have the following improvement:

$$
t_{n+2} \geq M_{1} \times t_{n+1}+M_{2} \times t_{n}
$$

where $M_{1}=N_{1}$, and $M_{2}=N_{2}-\left(N_{1} \times M_{1}\right)$. The matrices $M_{1}$ and $M_{2}$ are listed in the Appendix. The asymptotic rate of growth of the lower bound for $\mathrm{cfhc}\left(\mathrm{TS}_{n}\right)$ as determined by (2.2) is $c \times r_{2}^{n}$, where $r_{2}$ is the dominant eigenvalue of the 12 by 12 matrix $P_{2}$, where

$$
P_{2}=\left[\begin{array}{cc}
M_{1} & M_{2} \\
I_{6} & 0
\end{array}\right],
$$

and where $I_{6}$ is the 6 by 6 identity matrix. The value of $r_{2}$ is (to four decimal places) 2.1215 .

2.2.3. Case $k \geq 3$ : creating cfhc's of $\mathrm{TS}_{n+k}$ from cfhc's of $\mathrm{TS}_{n}$. We now show how to enumerate the ways in which cfhc's of TS $_{n+k}$ can be created from cfhc's of $\mathrm{TS}_{n}$, without having to draw figures corresponding to those shown in Figs. 5 and 6.

A cfhc of $\mathrm{TS}_{n+k}$ is created by taking a drawing of $\mathrm{TS}_{n+k}$, placing a cfhc of $\mathrm{TS}_{n}$ on vertices 1 to $n$, removing one or two of its outermost edges, placing a cfhe of $\mathrm{TS}_{3+k}$ on vertices $n-2$ to $n+k$, and then removing one or two of the 
latter cfhc's innermost edges, so that each of the edges $(n-2, n-1),(n-1, n)$, and $(n-2)$ will have been removed from either the former or latter cfhc. For example, Fig. 7 shows how a cfhc of $\mathrm{TS}_{11}$ is created by drawing a $\gamma(X, 8)$ on vertices 1 to 8 , removing the edge $X=(6,7)$, drawing a $\gamma(y z, 6)$ on vertices 6 to 11 , and removing the edges $y=(7,8)$ and $z=(6,8)$. The following is a summary of all ways in which cfhc's of $\mathrm{TS}_{n+k}$ can be thus created:

\begin{tabular}{cccc}
\hline $\begin{array}{c}\text { Cfhc on vertices } \\
\text { 1 to } n\end{array}$ & $\begin{array}{c}\text { Edge(s) removed } \\
\text { from cfhc } \\
\text { on } 1 \ldots n\end{array}$ & $\begin{array}{c}\text { Cfhc on vertices } \\
n-2 \text { to } n+k\end{array}$ & $\begin{array}{c}\text { Edge(s) removed } \\
\text { from cfhc } \\
\text { on } n-2 \ldots n+k\end{array}$ \\
\hline$\gamma(X, n)$ & $X$ & $\gamma(y z, 3+k)$ & $y, z$ \\
$\gamma(Y, n)$ & $Y$ & $\gamma(z x, 3+k)$ & $z, x$ \\
$\gamma(Z, n)$ & $Z$ & $\gamma(x y, 3+k)$ & $x, y$ \\
$\gamma(Y Z, n)$ & $Y$ & $\gamma(z x, 3+k)$ & $z, x$ \\
$\gamma(Y Z, n)$ & $Z$ & $\gamma(x y, 3+k)$ & $x, y$ \\
$\gamma(Y Z, n)$ & $Y, Z$ & $\gamma(x, 3+k)$ & $x$ \\
$\gamma(Z X, n)$ & $Z$ & $\gamma(x y, 3+k)$ & $x, y$ \\
$\gamma(Z X, n)$ & $X$ & $\gamma(y z, 3+k)$ & $y, z$ \\
$\gamma(Z X, n)$ & $Z, X$ & $\gamma(y, 3+k)$ & $y, z$ \\
$\gamma(X Y, n)$ & $X$ & $\gamma(y z, 3+k)$ & $z, x$ \\
$\gamma(X Y, n)$ & $Y$ & $\gamma(z x, 3+k)$ & $z$ \\
$\gamma(X Y, n)$ & $X, Y$ & $\gamma(z, 3+k)$ & \\
\hline
\end{tabular}

For $\Omega=X, Y, Z, Y Z, Z X, X Y$ and $\alpha=x, y, z, y z, z x, x y$, let $T_{n}(\Omega, \alpha)$ be the number of $\gamma(\Omega, \alpha)$ of $\operatorname{TS}_{n}$. Then the following inequality follows from the above summary:

$$
\begin{aligned}
t_{n+k}(\Omega) \geq & T_{3+k}(\Omega, y z) \times t_{n}(X) \\
& +T_{3+k}(\Omega, z x) \times t_{n}(Y) \\
& +T_{3+k}(\Omega, x y) \times t_{n}(Z) \\
& +\left(T_{3+k}(\Omega, z x)+T_{3+k}(\Omega, x y)+T_{3+k}(\Omega, x)\right) \times t_{n}(Y Z) \\
& +\left(T_{3+k}(\Omega, x y)+T_{3+k}(\Omega, y z)+T_{3+k}(\Omega, y)\right) \times t_{n}(Z X) \\
& +\left(T_{3+k}(\Omega, y z)+T_{3+k}(\Omega, z x)+T_{3+k}(\Omega, z)\right) \times t_{n}(X Y) .
\end{aligned}
$$

Let $T_{n}$ be the 6 by 6 matrix whose entries are $T_{n}(\Omega, \alpha)$, and let

$$
Q=\left[\begin{array}{llllll}
0 & 0 & 0 & 1 & 0 & 0 \\
0 & 0 & 0 & 0 & 1 & 0 \\
0 & 0 & 0 & 0 & 0 & 1 \\
1 & 0 & 0 & 0 & 1 & 1 \\
0 & 1 & 0 & 1 & 0 & 1 \\
0 & 0 & 1 & 1 & 1 & 0
\end{array}\right]
$$




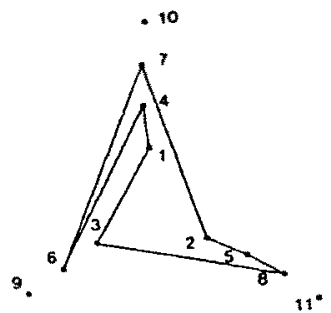

$\gamma(\mathrm{X}, 8)$

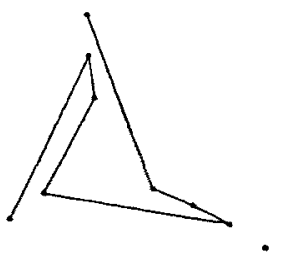

$\gamma(\mathrm{X}, 8)$ minus edge $\mathrm{X}$

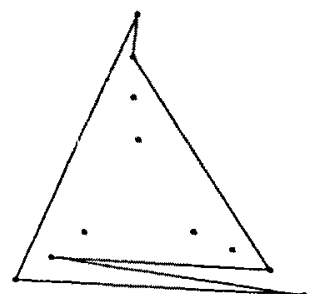

$\gamma(y \mathrm{z}, 6)$

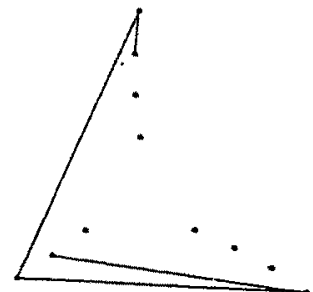

$\gamma(y z, 6)$ minus edges $y$ and $z$

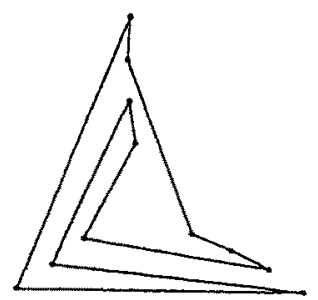

Fig. 7. Creating a cfhe of $\mathrm{TS}_{11}$ from a cfhe of $\mathrm{TS}_{8}$ and a cfhc of $\mathrm{TS}_{6}$.

Then the preceding inequality can be written in matrix form, namely

$$
t_{n+k} \geq N_{k} \times t_{n}, \quad \text { where } \quad N_{k}=T_{3+k} \times Q .
$$

As before, we can improve slightly on this inequality by creating cfhc's of $\mathrm{TS}_{n+k}$ from cfhc's of $\mathrm{TS}_{n}, \mathrm{TS}_{n+1}, \ldots, \mathrm{TS}_{n+k-1}$ (i.e., not just from $\mathrm{TS}_{n}$ ). This yields the following:

$$
t_{n+k} \geq M_{1} \times t_{n+k-1}+M_{2} \times t_{n+k-2}+\cdots+M_{k} \times t_{n}
$$

where

$$
M_{k}=N_{k}-\left(N_{1} \times M_{k-1}+N_{2} \times M_{k-2}+\cdots+N_{k-1} \times M_{1}\right)
$$

The matrices $T_{3+k}$ were determined by computer enumeration, for $k=3$ to 11 . 
Programs were written in $C$ and run on a Vax 11-750 with operating system Unix 4.2. Time constraints prevented further computations. For successive values of $k$, the amount of c.p.u. time used increased by a factor of about 6. As approximately 50 hours of c.p.u. time were required for $k=11$, about 300 hours might be necessary for the case $k=12$.

As was the case with (2.2), the inequality (k.2) can be written as an inequality involving the single $6 k$ by $6 k$ matrix $P_{k}$, where

$$
P_{k}=\left[\begin{array}{ccccc}
M_{1} & M_{2} & \cdots & M_{k-1} & M_{k} \\
I_{6} & 0 & \cdots & 0 & 0 \\
0 & I_{6} & \cdots & 0 & 0 \\
\cdots & \ldots & \cdots & \ldots & \ldots \\
0 & 0 & \cdots & I_{6} & 0
\end{array}\right]
$$

The dominant eigenvalue $r_{k}$ of $P_{k}$ gives the asymptotic rate of growth of the lower bound for cfhc (TS ${ }_{n}$ ) as determined by (k.2). The matrices $T_{3+k}$ and the eigenvalues $r_{k}$ are all given in the Appendix. The best lower bound (to four decimal places), achieved with $k=11$, is $\mathrm{cfhc}\left(\mathrm{TS}_{n}\right) \geq c \times 3.2684^{n}$. Thus it follows immediately that $\bar{\Phi}(n) \geq c \times 3.2684^{n}$.

\section{Open Problems}

We have established an improved lower bound for $\bar{\Phi}(n)$, namely

$$
c \times 3.2684^{n} \leq \bar{\Phi}(n), \quad \text { for some constant } c \text {, }
$$

by counting only a proper subset of the cfhc's of $\mathrm{TS}_{n}$. Thus determining $\mathrm{cfhc}\left(\mathrm{TS}_{n}\right)$ explicitly or even asymptotically is still open. Extrapolating the values $r_{k}$ (see the Appendix) suggests that $\mathrm{cfhc}\left(\mathrm{TS}_{n}\right)$ might be something near $c \times 3.5^{n}$, for some constant $c$.

There are several rectilinear drawings of $K_{n}$ that have fewer crossings than $\mathrm{TS}_{n}$, and almost certainly have more cfhc's (see $[\mathrm{H}]$ ). $\mathrm{TS}_{n}$ was selected for analysis of its number of cfhc's because its symmetries allow for a recursive counting argument. Crucial to our argument is the fact that any $k$ consecutive vertices of $\mathrm{TS}_{n}$ induce a drawing isomorphic to $\mathrm{TS}_{k}$; we know of no drawing of $K_{n}$ with fewer crossings than $\operatorname{TS}_{n}$ which has this property.

For all values of $\boldsymbol{n}$ for which cfhc's have been explicitly counted, no drawing of $\mathrm{TS}_{n}$ has more cfhc's than a certain non-rectilinear drawing $B K_{n}$ (see $[\mathrm{H}]$ ); all values of lower bounds for $\Phi(n)$ which appear in the table in Section 1, correspond to the number of cfhc's of $B K_{n}$. We conjecture that the number of cfhc's of $B K_{n}$ serve as an upper bound for both $\bar{\Phi}(n)$ and $\Phi(n)$. From the values of cfhc $\left(B K_{n}\right)$ that appear in this table, we conjecture that cfhc $\left(B K_{n}\right)$ is asymptotically $c \times r^{n}$, where $4.3<r<4.5$. Finally, we conjecture that both $\bar{\Phi}(n)$ and $\Phi(n)$ are less than $c \times 4.5^{n}$, for some constant $c$. 


\section{Acknowledgments}

I would like to thank Selim Akl, Jon Davis, David Gregory, and Peter Taylor for the many insightful comments and fruitful suggestions which they offered and which contributed to the development of the ideas in this paper. I am especially indebted to Selim Akl and Peter Taylor, with whom I was in frequent consultation when the original version of this paper was written. Finally, I thank Dave Rappaport and the McGill School of Computer Science Computational Geometry Laboratory for providing the computer facilities used to draw the figures.

\section{Appendix}

\section{This Appendix contains}

the matrices $T_{6}$ to $T_{14}$ (augmented),

the dominant eigenvalues of matrices $P_{1}$ to $P_{11}$.

Recall that in a cfhc of the drawing $\mathrm{TS}_{n}$,

$X, Y$, and $Z$ represent, respectively, the edges $(n-2, n-1),(n-1, n)$, and $(n-2, n)$,

$x, y$, and $z$ represent, respectively, the edges $(1,2),(2,3)$, and $(1,3)$.

Recall that the entry $t_{n}(\Omega, \alpha)$ of matrix $T_{n}$ is the number of cfhc's of the drawing TS $_{n}$ with outermost edge set $\Omega$ and innermost edge set $\alpha$, where $\Omega$ takes on the values $X, Y, X, Y Z, Z X, X Y$ and $\alpha$ takes on the values $x, y, z, y z, z x, x y$. For example, the entry in row 5 , column 2 of matrix $T_{7}$ is the number of cfhc's of $\mathrm{TS}_{7}$ with outermost edge set $\{Z, X\}$ and innermost edge set $\{y\}$, i.e., the number of cfhc's of TS $_{7}$ that contain edges $(5,7)$ and $(5,6)$ but not edge $(6,7)$, and that contain edge $(2,3)$ but not edges $(1,2)$ and $(1,3)$. For the sake of completeness, the matrices $T_{n}$ have been augmented in this Appendix to include a seventh row, corresponding to those cfhc's containing none of the edges $X, Y$, and $Z$, and a seventh column, corresponding to those cf hc's containing none of the edges $x, y$, and $z$. Thus, for $n>3$, the sum of all entries of the augmented matrix $T_{n}$ gives the total number of cfhc's of the drawing TS $_{n}$.

Recall that the number of different cfhc's of the drawing $\mathrm{TS}_{n+k}$ that can be created by adding exactly $k$ vertices outside a drawing of $\operatorname{TS}_{n}$ is given by the equation

$$
t_{n+k} \geq N_{k} \times t_{n} \text {, }
$$

where $N_{k}=T_{3+k} \times Q$, and where the entries of the column vector $t_{n}$ are the number 
of cfhc's of $\mathrm{TS}_{n}$ with outermost edge set (respectively) $X, Y, Z, Y Z, Z X, X Y$. The matrix $Q$ is shown below.

Recall that the number of different cfhc's of the drawing TS $_{n+k}$ that can be created by adding $1,2, \ldots$, or $k$ vertices to the drawings $\mathrm{TS}_{n+k-1}$, $\mathrm{TS}_{n+k-2}, \ldots, \mathrm{TS}_{n}$, respectively, is given by the equation

$$
t_{n+k} \geq M_{1} \times t_{n+k-1}+M_{2} \times t_{n+k-2}+\cdots+M_{k} \times t_{n},
$$

where $M_{k}=N_{k}-\left(N_{1} \times M_{k-1}+N_{2} \times M_{k-2}+\cdots+N_{k-1} \times M_{1}\right)$.

Recall that the asymptotic rate of growth of the number of cfhc's of TS $_{n}$ determined by equation (k.2) is equal to $c \times r_{k}^{n}$, where $r_{k}$ is the dominant eigenvalue of the $6 k$ by $6 k$ matrix $P_{k}$, shown below.

Recall that the dominant eigenvalue of $P_{k}$ is the largest real root of the characteristic polynomial of $P_{k}$.

$$
Q=\left[\begin{array}{cccccc}
0 & 0 & 0 & 1 & 0 & 0 \\
0 & 0 & 0 & 0 & 1 & 0 \\
0 & 0 & 0 & 0 & 0 & 1 \\
1 & 0 & 0 & 0 & 1 & 1 \\
0 & 1 & 0 & 1 & 0 & 1 \\
0 & 0 & 1 & 1 & 1 & 0
\end{array}\right] . \quad P_{k}=\left[\begin{array}{ccccc}
M_{1} & M_{2} & \cdots & M_{k-1} & M_{k} \\
I_{6} & 0 & \cdots & 0 & 0 \\
0 & I_{6} & \cdots & 0 & 0 \\
\cdots & \cdots & \cdots & \cdots & \cdots \\
0 & 0 & \cdots & I_{6} & 0
\end{array}\right]
$$

\begin{tabular}{|c|c|c|c|c|c|c|}
\hline 2 & 1 & 1 & 0 & 0 & 0 & 0 \\
\hline 1 & 2 & 1 & 0 & 0 & 0 & 0 \\
\hline 1 & 1 & 2 & 0 & 0 & 0 & 0 \\
\hline 0 & 0 & 0 & 1 & 2 & 2 & 0 \\
\hline 0 & 0 & 0 & 2 & 1 & 2 & 0 \\
\hline 0 & 0 & 0 & 2 & 2 & 1 & 0 \\
\hline 0 & 0 & 0 & 0 & 0 & 0 & 2 \\
\hline 2 & 4 & 2 & 1 & 2 & 1 & 1 \\
\hline 3 & 2 & 4 & 2 & 1 & 2 & 1 \\
\hline 4 & 2 & 3 & 2 & 1 & 2 & 2 \\
\hline 1 & 2 & 1 & 3 & 4 & 3 & 0 \\
\hline 2 & 1 & 2 & 3 & 3 & 2 & 0 \\
\hline 2 & 1 & 2 & 1 & 3 & 3 & 0 \\
\hline 2 & 1 & 1 & 0 & 0 & 0 & 4 \\
\hline 10 & 7 & 12 & 5 & 5 & 7 & 5 \\
\hline 14 & 10 & 10 & 7 & 6 & 6 & 6 \\
\hline 10 & 10 & 7 & 5 & 7 & 5 & 7 \\
\hline 6 & 5 & 7 & 6 & 8 & 8 & 1 \\
\hline 7 & 5 & 5 & 3 & 7 & 6 & 1 \\
\hline 6 & 7 & 5 & 7 & 8 & 8 & 2 \\
\hline 6 & 7 & 5 & 1 & 2 & 1 & 12 \\
\hline 38 & 36 & 36 & 26 & 22 & 22 & 24 \\
\hline 36 & 38 & 36 & 22 & 26 & 22 & 24 \\
\hline
\end{tabular}

Matrices $T_{6}$ to $T_{14}$ (augmented) 
Matrices $T_{6}$ to $T_{14}$ (augmented)-continued

\begin{tabular}{|c|c|c|c|c|c|c|}
\hline 36 & 36 & 38 & 22 & 22 & 26 & 24 \\
\hline 26 & 22 & 22 & 20 & 22 & 22 & 9 \\
\hline 22 & 26 & 22 & 22 & 20 & 22 & 9 \\
\hline 22 & 22 & 26 & 22 & 22 & 20 & 9 \\
\hline 24 & 24 & 24 & 9 & 9 & 9 & 48 \\
\hline 130 & 118 & 121 & 68 & 82 & 70 & 96 \\
\hline 145 & 121 & 141 & 75 & 84 & 86 & 94 \\
\hline 137 & 130 & 145 & 90 & 84 & 79 & 99 \\
\hline 84 & 82 & 84 & 64 & 70 & 64 & 42 \\
\hline 79 & 70 & 86 & 58 & 64 & 53 & 41 \\
\hline 90 & 68 & 75 & 47 & 64 & 58 & 42 \\
\hline 99 & 96 & 94 & 42 & 42 & 41 & 160 \\
\hline 526 & 465 & 489 & 257 & 282 & 300 & 393 \\
\hline 558 & 534 & 526 & 321 & 315 & 319 & 394 \\
\hline 534 & 441 & 465 & 245 & 300 & 271 & 393 \\
\hline 319 & 271 & 300 & 186 & 218 & 208 & 178 \\
\hline 321 & 245 & 257 & 139 & 196 & 186 & 175 \\
\hline 315 & 300 & 282 & 196 & 212 & 218 & 182 \\
\hline 394 & 393 & 393 & 175 & 182 & 178 & 580 \\
\hline 1990 & 2077 & 2077 & 1192 & 1108 & 1105 & 1650 \\
\hline 2077 & 1990 & 2077 & 1105 & 1192 & 1108 & 1650 \\
\hline 2077 & 2077 & 1990 & 1108 & 1105 & 1192 & 1650 \\
\hline 1192 & 1105 & 1108 & 703 & 745 & 745 & 754 \\
\hline 1108 & 1192 & 1105 & 745 & 703 & 745 & 754 \\
\hline 1105 & 1108 & 1192 & 745 & 745 & 703 & 754 \\
\hline 1650 & 1650 & 1650 & 754 & 754 & 754 & 2232 \\
\hline 7785 & 6920 & 7480 & 3658 & 4203 & 3771 & 6581 \\
\hline 8271 & 7480 & 7858 & 3992 & 4377 & 4289 & 6736 \\
\hline 7818 & 7785 & 8271 & 4396 & 4374 & 4081 & 6739 \\
\hline 4374 & 4203 & 4377 & 2553 & 2682 & 2556 & 3158 \\
\hline 4081 & 3771 & 4289 & 2365 & 2556 & 2252 & 3071 \\
\hline 4396 & 3658 & 3992 & 2154 & 2553 & 2365 & 3037 \\
\hline 6739 & 6581 & 6736 & 3037 & 3158 & 3071 & 8412 \\
\hline 31762 & 29365 & 29190 & 14537 & 15759 & 16227 & 27613 \\
\hline 32690 & 32079 & 31762 & 16754 & 16937 & 17041 & 28313 \\
\hline 32079 & 27782 & 29365 & 13976 & 16273 & 15341 & 27313 \\
\hline 17041 & 15341 & 16227 & 8699 & 9612 & 9210 & 12931 \\
\hline 16754 & 13976 & 14537 & 7418 & 8889 & 8699 & 12474 \\
\hline 16937 & 16273 & 15759 & 8889 & 9314 & 9612 & 12968 \\
\hline 28313 & 27313 & 27613 & 12474 & 12968 & 12931 & 33265 \\
\hline
\end{tabular}

Dominant eigenvalue of matrices $P_{1}$ to $P_{11}$

\begin{tabular}{ll}
1.8124 & 2.9551 \\
2.1215 & 3.0457 \\
2.4992 & 3.1410 \\
2.6004 & 3.2039 \\
2.7273 & 3.2684 \\
2.8726 & \\
\hline
\end{tabular}




\begin{tabular}{rr}
\multicolumn{2}{c}{ Number of cfhc's of $\mathrm{TS}_{3}$ to $\mathrm{TS}_{14}$} \\
\hline $\begin{array}{l}\text { With at least one innermost edge } \\
\text { and at least one outermost edge }\end{array}$ & $\begin{array}{c}\text { Total } \\
\text { number }\end{array}$ \\
\hline- & 1 \\
- & 3. \\
- & 8 \\
27 & 29 \\
79 & 91 \\
257 & 313 \\
942 & 1188 \\
3166 & 4154 \\
11517 & 15527 \\
45441 & 62097 \\
165986 & 233042 \\
642106 & 918595 \\
\hline
\end{tabular}

\section{References}

[A] S. Akl, A lower bound on the maximum number of crossing-free Hamilton cycles in a rectilinear drawing of $K_{n}$, Ars Combin. 7 (1979), 7-18.

[ACNS] M. Ajtai, V. Chvátal, M. Newborn, and E. Szemerédi, Crossing-free subgraphs, Ann. Discrete Math. 12 (1982), 9-12.

[EG] P. Erdos and R. K. Guy, Crossing number problems, Amer. Math. Monthly 80 (1973), 52-58.

[G] R. K. Guy, Unsolved problems, Amer. Math. Monthly 88 (1981), 757.

[H] R. B. Hayward, The Optimal Crossing-Free Hamilton Cycle Problem for Planar Drawings of the Complete Graph, M.Sc. thesis, Queen"s University, Kingston, Ontario, 1982.

[NM] M. Newborn and W. O. J. Moser, Optimal crossing-free Hamiltonian circuit drawings of $K_{n}, J$. Combin. Theory Ser. B 29 (1980), 13-26.

Received July 25, 1985, and in revised form March 17, 1986. 